\title{
FEATURES OF OCULAR INFESTATION BY TOXOCARA* BY
}

\section{M. DUGUID}

Department of Pathology, Institute of Ophthalmology, University of London

IN Britain, ocular involvement by Toxocara was first recognized by Ashton (1960), who reported four cases of granulomatous lesions of the retina due to this ascarid nematode, common in dogs and cats. Stimulated by these revelations, attention has since been focused on this aspect of ophthalmology and it appears that some ocular lesions caused by Toxocara have in the past been attributed mistakenly to other causes such as organized haemorrhage or exudate, Coats's disease, and choroiditis. In Ashton's series, all four eyes had been enucleated because the fundus lesions appeared similar to those of retinoblastoma, and not until after the completion of histological examination was the correct diagnosis established.

28 cases of ocular infestation by Toxocara have now been examined at the Institute of Ophthalmology, London:

\begin{tabular}{c|c|c|c}
\hline \multirow{2}{*}{ Total Number of Cases } & \multicolumn{2}{|c|}{ Histologically } & $\begin{array}{c}\text { Clinically Typical under } \\
\text { Observation }\end{array}$ \\
\cline { 2 - 3 } & Proved & Unproved but Typical & \\
\hline 28 & 13 & 6 & 9 \\
\hline
\end{tabular}

It is evident that the majority of these cases have been diagnosed only after enucleation and it is suggested that Toxocara infestation is more common than was formerly apparent.

Further study of these cases revealed several features suggestive of Toxocara infestation and these will now be considered more closely as this knowledge might obviate the necessity for enucleation in some future cases.

Contact with Dogs and/or Cats.-The incidence of Toxocara in these domestic animals, as judged from different writers, varies greatly in different parts of the world. Some of the figures given for foreign countries are:

13.5 per cent. of dogs in Marseilles (Solomon, 1933).

20 per cent. of dogs in Boston, U.S.A. (Heiner and Kevy, 1956).

21.8 per cent. of 174 dogs in Melbourne, Australia (Pullar, 1946).

$82 \cdot 8$ per cent. of 100 dogs in Calcutta (Maplestone and Bhaduri, 1940).

In the United Kingdom the figures are:

70.8 per cent. of dogs in Cambridge (Nuttall and Strickland, 1908).

Toxocara canis in $16 \cdot 3$ per cent. of dogs and Toxocara cati in 61.8 per cent. of cats in Wales (Lewis, 1927). 
The chances of coming in contact with an infested dog or cat are therefore not to be overlooked, and particularly is this so in children who are often seen cuddling these domestic pets. Moreover, yard or garden soil may become heavily fouled by the ova present in faeces from a dog with Toxocara, and herein lies the danger for a child of dirt-eating age. These children indiscriminately pass anything into the mouth and are liable to swallow ova which may be accidentally picked up while playing in the garden. Once embryonated ova are swallowed they are likely to hatch out in the intestine, liberating larvae which, according to Sprent (1954), are active borers and liable to migrate anywhere in the body, but especially to the lungs, liver, brain, and by chance to the eye. Considering that many children encounter these common pets it may seem superfluous to inquire about a history of contact, but it is surprising how often parents have helped by stating that a child (with a suspected Toxocara lesion) had been in contact with a puppy with worms.

Age Group Involved.-Until recently the eldest recorded case of ocular Toxocara infestation in this series was aged 16 years, but the majority of ocular lesions presented in children about 4 to 6 years of age. Such a distribution is not surprising considering the likelihood of ingesting Toxocara ova during the first few years of life, which may be followed by a latent period before the ocular nematode lesion presents.

Site of Lesion.-The granulomatous lesion is typically solitary and is situated posteriorly in close proximity to the optic disc and macula. Usually the granuloma

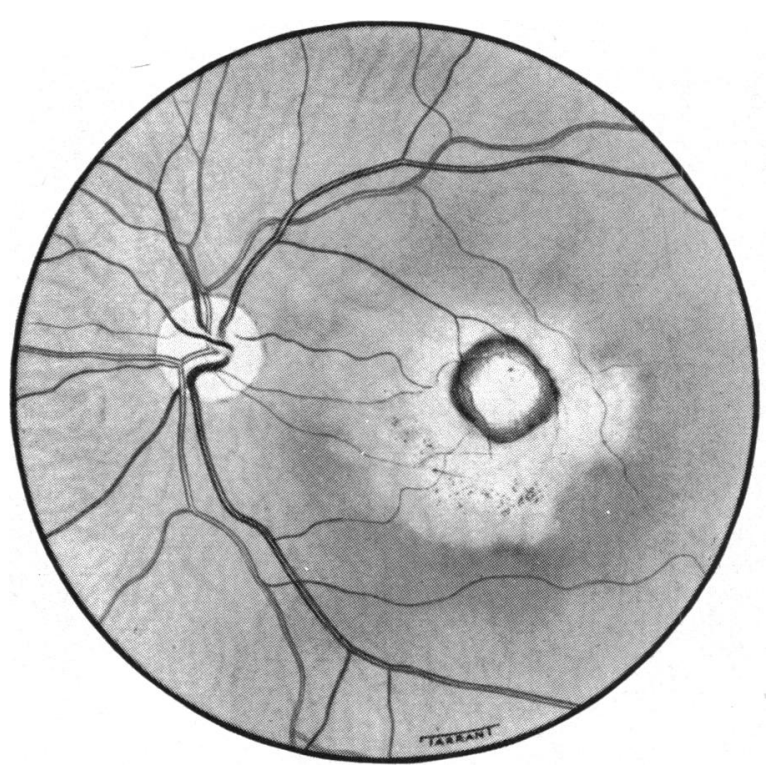
containing the larva has been located in the macular region or temporal to the optic disc (Fig. 1).

Fig. 1.-Location of larva.

On no occasion so far has the lesion been found either in the peripheral fundus or nasal to the optic disc. Only in cases of chronic endophthalmitis with retinal detachment due to Toxocara (Duguid, 1961) have larval fragments been located more anteriorly, and that merely because of the accompanying total 
retinal detachment. In some cases of larval granulomatosis with exudation, the granuloma containing the second stage Toxocara larva may project forwards into the vitreous, appearing as a white mass with vitreous bands running between the mass and retina (Fig. 2). Such a condition might represent an intermediate stage between the intra-retinal granuloma and that of chronic endophthalmitis with total retinal detachment.

FIG. 2.-Appearance in macular region of white larval mass with vitreous bands between the mass and retina.

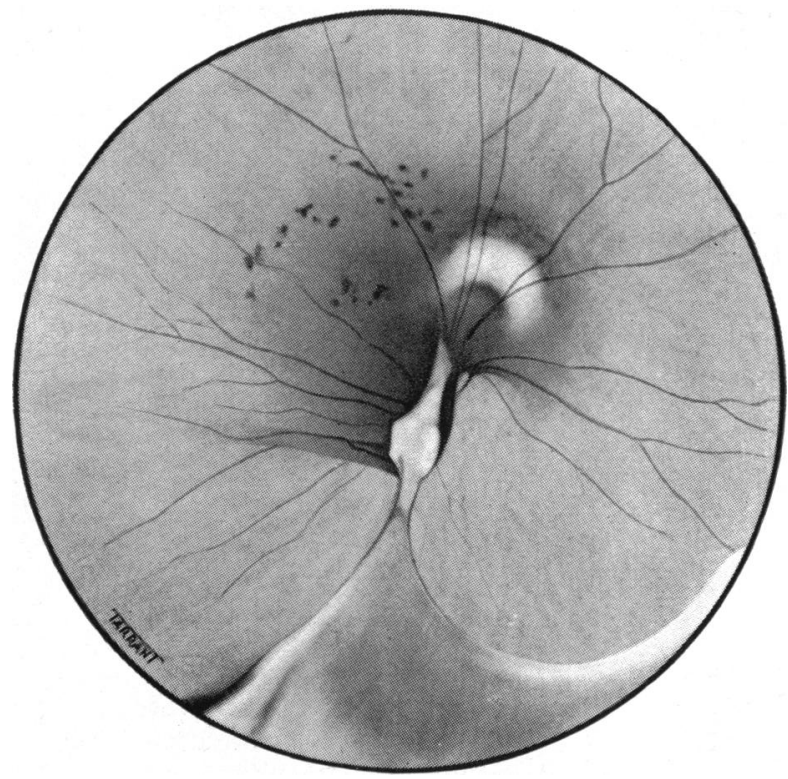

On two occasions the lesion actually implicated the temporal part of the optic disc: the fundus of one of these cases is shown in Fig. 3.

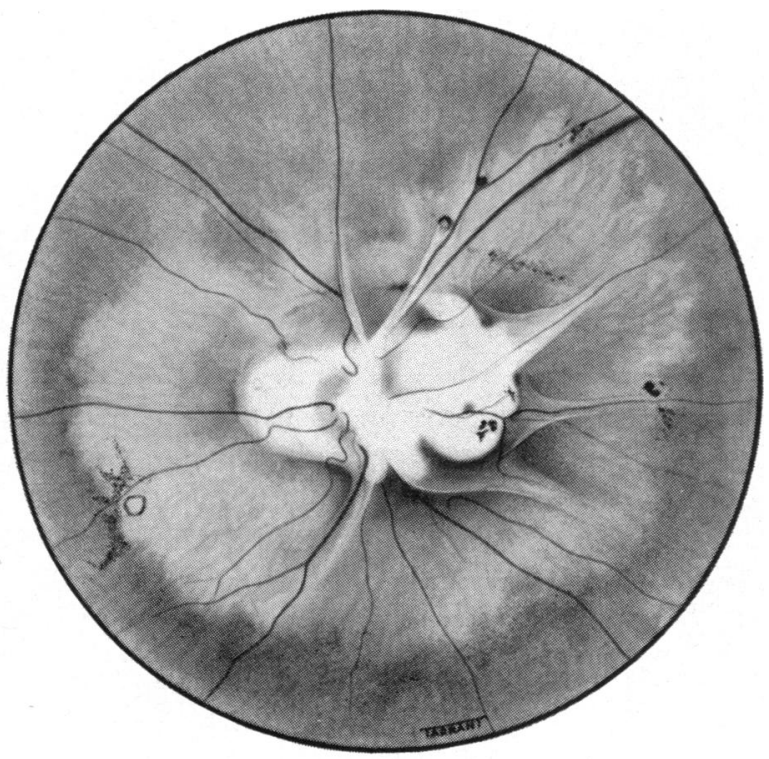

FIG. 3.-Lesion implicating temporal part of optic disc. 
Incidentally, in the series under review, the right and left eyes were equally involved.

The lesion is typically unilateral: bilateral ocular Toxocara infestation has not so far been encountered.

Colour and Nature of Lesion.-A Toxocara granuloma is typically white and its density depends on the amount of granulation tissue present. It is important to use not only the ophthalmoscope but also the slit lamp in looking for such variations of colour within the granuloma as are discernible in Fig. 4 (opposite), where a slightly darker area, crescentic in shape, is clearly visible. This darker area indicates the position of the larva within the granuloma, and such an area if found is highly suggestive and almost diagnostic of Toxocara. Fig. 5 shows the fundus of a girl with a Toxocara lesion in the macular area; here very little granulation tissue reaction has appeared and the larval outline is clearly visible. It might be mentioned in passing that this patient had been given tetracyclines orally for 3 days and the fundus lesion was then examined with ultra-violet light in the hope that the larva would selectively take up this drug, as in filariasis, and would then fluoresce on exposure to ultra-violet rays; disappointingly, no fluorescence was noted.

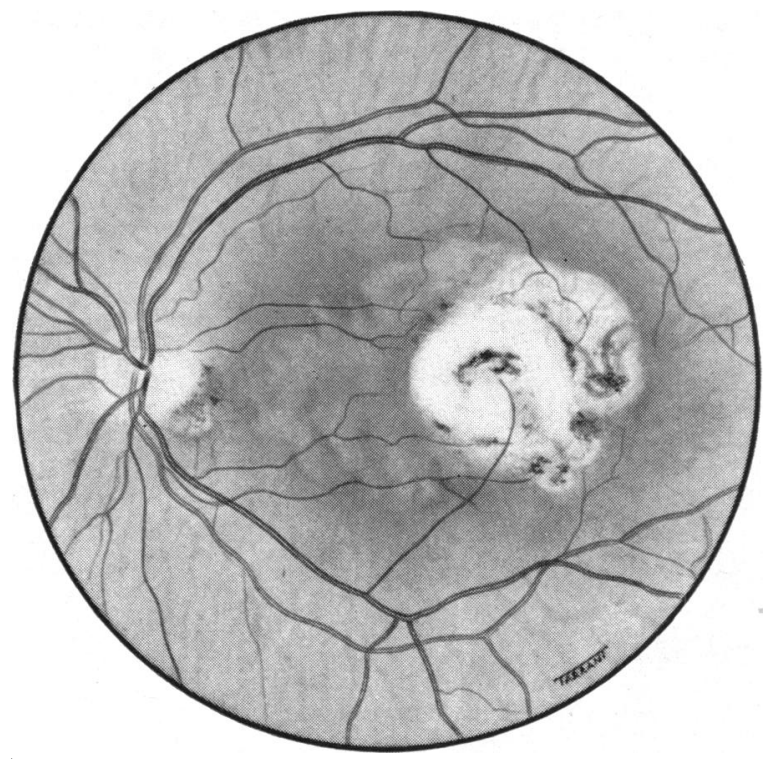

Fig. 5.-Lesion in macular area, showing larval outline.

Usually the granulomatous lesion has a round outline and a diameter about equal to that of the optic disc. The lesion is characteristically raised, and excites little surrounding pigmentary migration although some atrophy of the adjacent fundus is common. Tension lines or retinal folds radiating from the granuloma are frequently present and may be prominent (Fig. 6, overleaf). Particularly in lesions with an uneven surface, aberrant proliferative branches from nearby retinal vessels may be seen coursing across the flatter peripheral portion of the lesion before disappearing from view in the thicker centre as in the first case described by Ashton (1960). 
FEATURES OF OCULAR INFESTATION BY TOXOCARA

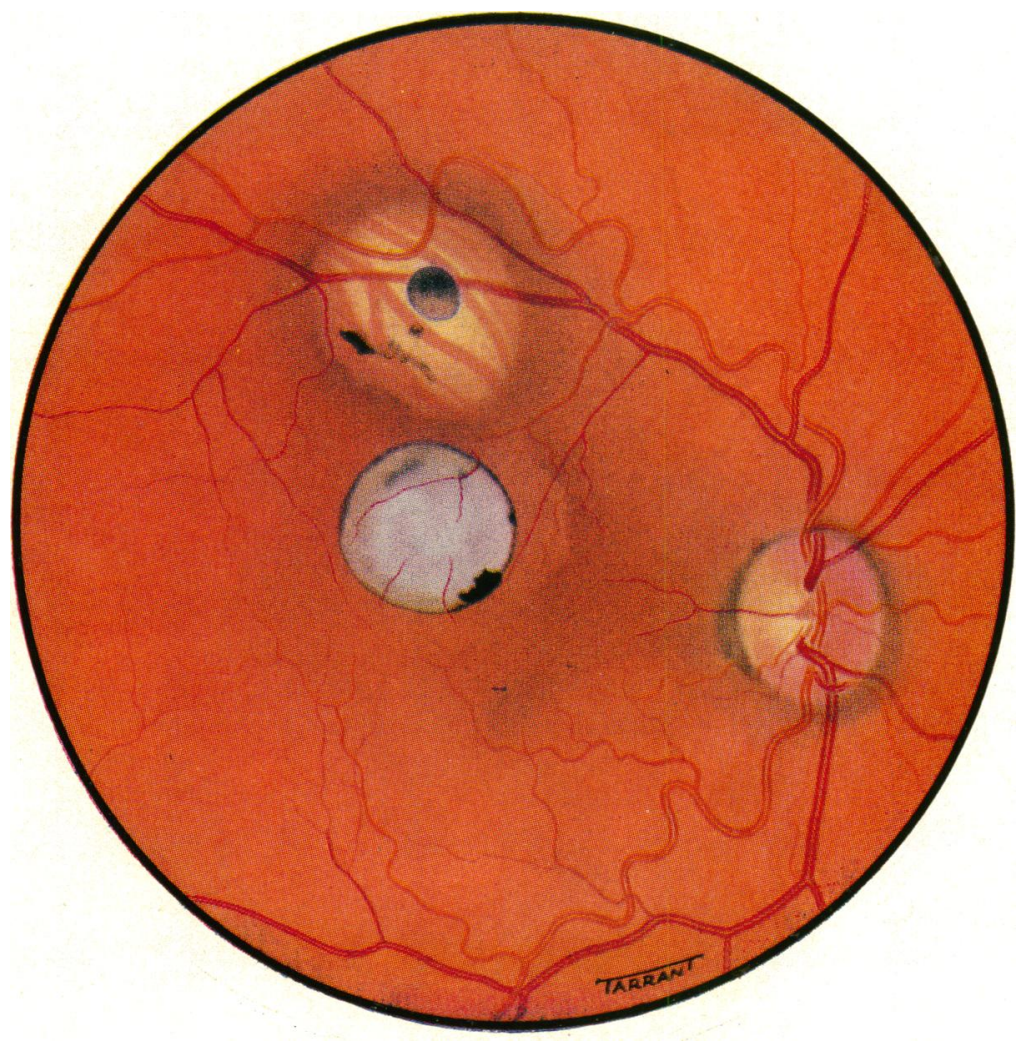

Fig. 4.-Variations of colour within the granuloma are readily visible.

To face page 792.] 


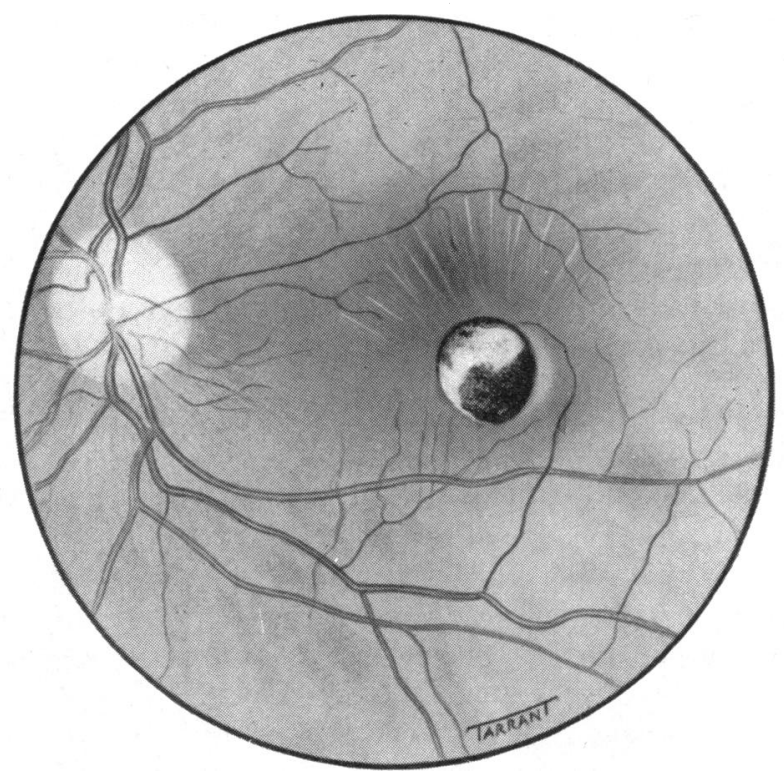

Fig. 6.-Tension lines radiating from granuloma.

Visual Acuity and Strabismus.-All cases from this series showed impaired visual acuity in the affected eye; in two cases the acuity had fallen only to $6 / 12$, but in the remainder a reduction of $6 / 60$ or even to counting fingers was common. In view of its preference for the posterior polar region, it is not surprising to find that the parasite causes such a marked decrease in visual acuity. Strabismus had been recorded on eight occasions and had been instrumental in first bringing the afflicted child to the ophthalmologist.

Chronic Endophthalmitis.-Perhaps more frequently present than the granulomatous lesion is a chronic endophthalmitis with retinal detachment due to Toxocara infestation, which should be suspected in all children presenting with unilateral ophthalmitis of apparently unknown aetiology. In addition-although it is too soon to be authoritative - it seems likely that many lesions hitherto diagnosed as Coats's disease will eventually prove to be caused by Toxocara. Before enucleation these eyes show no distinctive features which indicate the presence of an intra-ocular larva. Cases of chronic endophthalmitis with retinal detachment usually had a low-grade iritis with posterior synechiae to the lens and a cyclitic membrane between the detached retina and lens. Diagnosis in such patients is clinically difficult and one must often look for the other features of parasitic infestation mentioned in this article to assist with diagnosis.

Radiography.-This aid to diagnosis has been helpful in that no intra-ocular calcification has been observed about the site of the ocular nematode lesion-a feature which may be of value in differentiation from cases of retinoblastoma, about 75 per cent. of which contain calcified foci. In all the ocular histological sections examined, the absence of calcification in the Toxocara lesions is noteworthy, although it is probable that this might develop in long-standing cases. 
Laboratory Diagnosis.-Until the middle of 1960, the eosinophil count was the most important laboratory aid to diagnosis. An eosinophilia, usually 3 to 12 per cent., was present in most of the cases seen at the Institute of Ophthalmology. The degree of eosinophilia, according to Smith and Beaver (1953), is apparently proportionate to the severity of the infestation.

Search for ova and worms in the stools of persons with ocular Toxocara lesions has consistently been negative. This is not surprising considering that in the human that parasite does not develop beyond the second-stage larva. Thus, apart from histological examination of the enucleated eye with isolation of the causal larva, laboratory diagnosis has not previously proved of great help and an attempt was therefore made to evolve a suitable diagnostic test. Fresh adult Toxocara worms were obtained and were opened longitudinally to liberate their contained ova. The ova were incubated in 0.5 per cent. formol saline at $37^{\circ} \mathrm{C}$. for 1 to 2 weeks to promote growth into first-stage larvae. These larvae were then removed from the formol saline and washed in normal saline before being added to adult worms which had meanwhile been stored at $-30^{\circ} \mathrm{C}$. The worms and larvae were exposed to ultra-violet light for 5 hours before being frozen to $-70^{\circ} \mathrm{C}$. for one hour in a Pyrex tube surrounded by a snow and acetone mixture. The contents of the tube were then heated to $56^{\circ} \mathrm{C}$. for one hour in a water bath. By carrying out this sequence the arrest of further development of any viable ova or larva was fairly certain. The larvae and worms treated in this way were ground in a Tenbroeck tissue grinder with a little ether to give a fat-free antigen, before being added to sterile 0.5 per cent. phenol-saline to make a 1 per cent. solution, and buffered to neutral. The solution was then cultured to verify its sterility before being stored in ampoules at $-30^{\circ} \mathrm{C}$. About the middle of 1960 , healthy rabbits were inoculated with 5 to $10 \mathrm{ml}$. normal saline solution containing 200 live Toxocara larvae per $\mathrm{ml}$.; $0.1 \mathrm{ml}$. of the antigen prepared in this way was injected intradermally into healthy rabbits and into rabbits inoculated with live Toxocara larvae one month previously. All infested rabbits gave positive skin reactions consisting of erythema and induration around the site of the injected antigen, while the normal rabbits gave no cutaneous response whatsoever.

Following these results, one felt justified in trying this test on humans with ocular lesions thought to be due to Toxocara; $0.15 \mathrm{ml}$. of the antigen was injected intradermally in one forearm and the control into the other. The result was read 36 to 48 hours later and interpreted as for the Mantoux test. To date, this test has been carried out on twelve patients with ocular Toxocara lesions and in every instance a positive result has been obtained.

On eight occasions this antigen has been used on normal persons or patients with uveitis due to causes other than Toxocara and in every case the result has been negative. Unfortunately, due to shortage of antigen, it has so far been impossible to carry out the test on sufficient cases with lesions due to other parasitic or non-parasitic causes and it is too soon, therefore, to give any final opinion on the specificity of the test. Sprent and English (1958) prepared an antigen from live adult Toxocara canis worms, which were ground to a pulp, frozen at $-70^{\circ} \mathrm{C}$., and lyophilized. Their antigen, however, was used in conjunction with cutaneous scarification and the result was interpreted within the succeeding 20 minutes. Sprent and English expressed concern over the possibility of injecting live larvae, 
but with the more elaborately prepared antigen described above, this possibility is remote or non-existent.

Further comment on the test would obviously be unwise at this stage, but one can justly say that the test will prove helpful in establishing the diagnosis in doubtful cases.

Serological examination of patients with Toxocara infestation has been carried out by Kagan (1958), Sadun, Norman, and Allain (1957), and Jung and Pacheco (1960), but at this Institute no serological work on Toxocara has yet been started although it is hoped to begin in the near future.

Finally, there is the question of treatment. Initially this should be preventive; all domestic animals suspected of harbouring Toxocara (and this includes all puppies) should be seen by a veterinary surgeon with a view to initiating piperazine therapy as soon as possible. This drug is of value only for parasites within the intestinal canal and, therefore, would not be expected to help in established human ocular infestations, because the larva does not develop beyond the second-stage in man and therefore does not return to the intestine. It had been thought that photocoagulation might be employed (for the early granulomatous lesions with failure in vision) because coagulation of the parasite in situ might prevent the onset of chronic endophthalmitis with an inevitable secondary glaucoma and eventual enucleation. The necessity for this therapeutic attempt is doubtful. A case we have examined recently shows that the lesions may remain static for many years-if not indefinitely. Fig. 7 shows a fundus painting made 30 years ago of a probable Toxocara lesion which was published by Marshall (1931) as "exudative choroiditis" in a child aged 8 years. Fig. 4 (col. pl.) shows the same fundus in 1960, and comparison of these paintings reveals so little change that one would hesitate to embark upon heroic therapeutic measures.

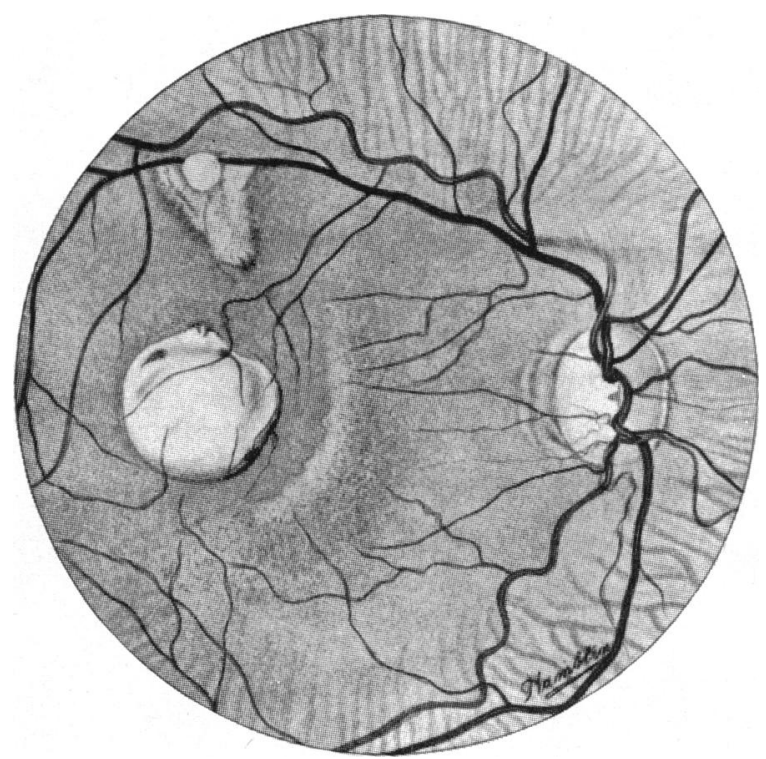

FIG. 7.-Appearance of fundus in 1930 of a child aged 8 (Marshall, 1931), for comparison with present state seen in Fig. 4.

By courtesy of the Honorary Editors of the Proceedings of the Royal Society of Medicine. 


\section{Summary}

(1) 28 cases of ocular Toxocara infestation have been examined.

(2) Two types of ocular lesion may be produced by the Toxocara larva: (a) retinal granuloma or $(b)$ chronic endophthalmitis.

(3) The clinical features of ocular Toxocara infestation are presented.

(4) A skin test designed to aid clinical diagnosis is described in detail.

(5) Possible methods of treatment are briefly discussed.

I am considerably indebted to Prof. Norman Ashton, not only for his valuable help in carrying out this work, but also for his criticism during the preparation of this article. My thanks are also due to Mr. J. E. M. Ayoub for kindly enabling me to observe some of the cases under his care and for his help in the preparation of this paper. It is a pleasure to acknowledge the technical assistance of Messrs. A. McNeil and P. Aldred in preparing the antigen, Miss E. FitzGerald for her excellent secretarial help, and the Medical Illustration Department of the Institute of Ophthalmology for the illustrations.

\section{REFERENCES}

Ashton, N. (1960). Brit. J. Ophthal., 44, 129.

DuguID, I. M. (1961). Ibid., 45, 705.

HeIner, D. C., and Kevy, S. V. (1956). New Engl. J. Med., 254, 629.

Jung, R. C., and Pacheco, G. (1960). Amer. J. trop. Med. Hyg., 9, 185.

KAGAN, I. G. (1958). J. Immunol. 80, 396.

LEWIS, E. A. (1927). J. Helminth., 5, 171.

MAPlestone, P. A., and BhADURI, N. V. (1940). Indian J. med. Res., 28, 595.

Marshall, J. C. (1931). Proc. roy. Soc. Med., 24, 607.

Nuttall, G. H. F., and Strickland, C. (1908). Parasitology, 1, 261.

Pullar, E. M. (1946). Aust. vet. J., 22, 85.

Sadun, E. H., Norman, L., and Allain, D. (1957). Amer. J. trop. Med. Hyg., 6, 562.

SMith, M. H. D., and BeAVer, P. C. (1953). Pediatrics, 12, 491.

Solomon, S. G. (1933). J. Helminth., 11, 157.

Sprent, J. F. A. (1954). J. Parasitol., 40, 608.

- and English, P. B. (1958). Aust. vet. J., 34, 161. 